\title{
OLD AND NEW GENERALIZATIONS OF LINE GRAPHS
}

\author{
JAY BAGGA
}

Received 8 October 2003

\begin{abstract}
Line graphs have been studied for over seventy years. In 1932, H. Whitney showed that for connected graphs, edge-isomorphism implies isomorphism except for $K_{3}$ and $K_{1,3}$. The line graph transformation is one of the most widely studied of all graph transformations. In its long history, the concept has been rediscovered several times, with different names such as derived graph, interchange graph, and edge-to-vertex dual. Line graphs can also be considered as intersection graphs. Several variations and generalizations of line graphs have been proposed and studied. These include the concepts of total graphs, path graphs, and others. In this brief survey we describe these and some more recent generalizations and extensions including super line graphs and triangle graphs.
\end{abstract}

2000 Mathematics Subject Classification: 05C75, 05C45, 05C62, 05C40.

1. Introduction. The line graph $L(G)$ of a graph $G$ is defined to have as its vertices the edges of $G$, with two being adjacent if the corresponding edges share a vertex in $G$. Line graphs have a rich history. The name line graph was first used by Harary and Norman [17] in 1960. But line graphs were the subject of investigation as far back as 1932 in Whitney's paper [24], where he studied edge isomorphism and showed that for connected graphs, edge-isomorphism implies isomorphism except for $K_{3}$ and $K_{1,3}$. The first characterization (partition into complete subgraphs) was given by Krausz [19].

Since this is a survey on generalizations of line graphs, we will not describe line graphs and their properties in any detail here. Instead, we refer the interested reader to a somewhat older but still an excellent survey on line graphs and line digraphs by Hemminger and Beineke [18]. A more recent book by Prisner [22] describes many interesting generalizations of line graphs. For general graph theoretic concepts and terminology not defined here, please see $[9,16]$.

In the rest of this section, we will make some general remarks on the nature of research in line graphs and the generalizations that have been studied. In the following sections, we describe some generalizations and variations of the line graph concept. We describe two, super line graphs and triangle graphs, in somewhat greater detail. We also mention some open problems in this area.

According to the above-mentioned article by Hemminger and Beineke, much of the effort in the early research concentrated on the determination problem (determine which graphs have a given graph as their line graph) and the characterization problem (characterize those graphs that are line graphs of some graph). Krausz [19] gave a characterization of line graphs in terms of complete subgraphs. There are several other characterizations mentioned in [18], including the forbidden subgraph characterization 


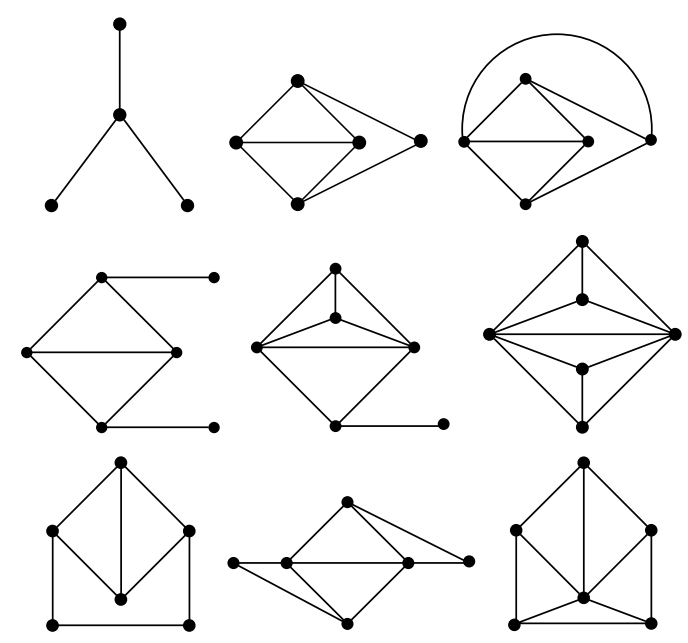

FIGURE 1.1

by Beineke [11], which states that a graph is a line graph if and only if it has no induced subgraph isomorphic to any of the graphs shown in Figure 1.1.

One can view the line graph as a transformation $G \rightarrow L(G)$. Repeated applications of this transformation yield iterated line graphs, which have been studied by several authors.

Thus iterated line graphs are defined by $L^{1}(G)=L(G)$ and $L^{n+1}(G)=L\left(L^{n}(G)\right.$ ) (provided that $L^{n}(G)$ is not null). Questions about convergence have been considered. If $G$ is the path $P_{n}$ on $n$ vertices, then $L(G)=P_{n-1}$. Hence $\left\{L^{n}\right\}$ terminates for paths. If $G$ is a cycle, then $L(G) \simeq G$. Also $L\left(K_{1,3}\right)=K_{3}$. Hence for these graphs $\left\{L^{n}\right\}$ becomes a constant. For all other connected graphs, $\left\{L^{n}\right\}$ contains arbitrarily large graphs [23]. Other properties of iterated line graphs such as Hamiltonicity have also been studied.

THEOREM 1.1 [13]. Let $G$ be a connected graph of order $p$ that is not a path. Then $L^{n}(G)$ is Hamiltonian for all $n \geq p-3$.

See Prisner [22] for details on the line graph transformation and several other similar concepts and results on iterations.

Line graphs have edges as their vertices. An edge can be viewed as a subset of two adjacent vertices, a clique of order two, a path of length one, or a subgraph of size one, among others. Depending on which view is considered, generalizations and extensions of line graphs result by generalizing the corresponding view. For example, in Section 4, we will describe a recent generalization called the triangle graph for which the vertex set consists of the triangles of $G$, with two being adjacent if they share an edge.

In the next section, we describe some earlier generalizations of line graphs.

\section{Old generalizations}

2.1. Total graphs. The total graph $T(G)$ of a graph $G$, defined by Behzad and Chartrand [10], takes both the vertices and edges of $G$ as elements for its set of vertices. 


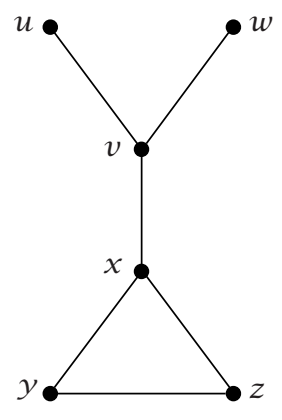

G

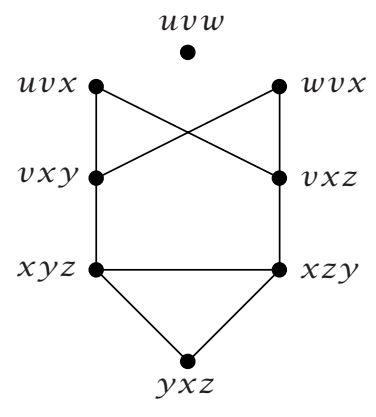

$P_{2}(G)$

FIGURE 2.1

Two vertices are adjacent if the corresponding elements of $G$ are either adjacent or incident.

Recall Vizing's theorem, which states that if $G$ has maximum degree $\Delta$, then $\chi(L(G))=$ $\Delta$ or $\Delta+1$, Behzad conjectured that $\chi(T(G))=\Delta+1$ or $\Delta+2$. Whether any graph has total chromatic number greater than $\Delta+2$ is still an open question [15].

2.2. Middle graphs. The middle graph $\operatorname{mid}(G)$ of $G$ is obtained from $G$ by inserting a new vertex into every edge of $G$ and by joining those pairs of new vertices which lie on adjacent edges of $G$. Thus $\operatorname{mid}(G)$ can also be considered as the intersection graph of all $K_{1}$ 's and $K_{2}$ 's in $G$ [22].

We observe that

(i) $L(G)$ is an induced subgraph of $\operatorname{mid}(G)$,

(ii) the subdivision graph $S(G)$ is a subgraph of $\operatorname{mid}(G)$,

(iii) $\operatorname{mid}(G)$ is a subgraph of $T(G)$.

2.3. Entire graphs. The entire graph $e(G)$ of a plane graph $G$ is the graph whose vertices correspond to vertices, edges, and regions of $G$. Two vertices of $e(G)$ are adjacent if the corresponding elements of $G$ are adjacent or incident. See [20] for some results on entire graphs.

2.4. Path graphs. The $r$ th path graph $\mathscr{P}_{r}(G)$ [12] has the paths of length $r$ in $G$ as its vertices and two such paths are adjacent if their union is a path or cycle of length $r+1$. For $r=1$, we obtain the line graph. An example for $r=2$ is shown in Figure 2.1. We observe that a path of length $r$ in $G$ corresponds to an isolated vertex in $\mathscr{P}_{r}(G)$ if and only if its end vertices have degree 1 in $G$. Thus, connectedness of $G$ is not inherited by the $r t h$ path graph for $r>1$. Broersma and Hoede [12] also show that the numbers of vertices and edges in $\mathscr{P}_{2}(G)$ are $\sum_{v}\left(\begin{array}{c}\operatorname{deg}(v) \\ 2\end{array}\right)$ and $(1 / 2) \sum_{v}\left[(\operatorname{deg}(v)-1) \sum_{u \sim v}(\operatorname{deg}(u)-\right.$ $1)$ ], respectively (where $\sim$ denotes adjacency).

2.5. $r$-subgraph distance graphs. Let $\mathscr{S}_{r}(G)$ be the graph whose vertices are the subgraphs of $G$ having $r$ edges, with two such being adjacent if the symmetric difference of their edge sets consists precisely of two adjacent edges of $G$. $S_{r}(G)$ is called 


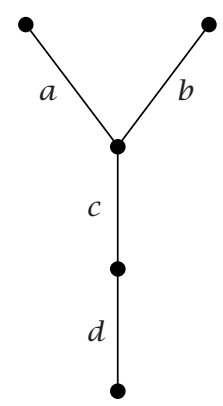

G

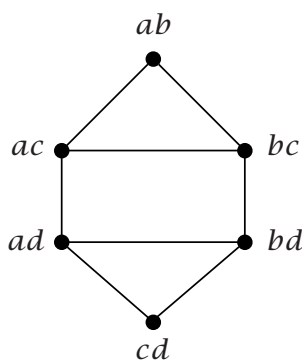

$S_{2}(G)$

FIGURE 2.2

the $r$-subgraph distance graph (Chartrand et al. [14]). An example for $r=2$ is shown in Figure 2.2.

Note that two subgraphs (with $r$ edges) in $G$ are adjacent if one can be obtained from the other by "pivoting" one edge. Because $\mathscr{S}_{r}(G)$ is connected whenever $G$ is connected (and has at least $r$ edges), it provides a measure of the distance between two subgraphs of size $r$ in $G$.

3. Super line graphs. In this section, we describe in somewhat greater detail a more recent generalization, the super line graph. Super line graphs were introduced by Bagga et al. in [6]. Since then, the study of super line graphs has progressed much further $[1,2,3,4,5,6]$.

3.1. Definition and basic properties. For a fixed integer $r$ (with $1 \leq r \leq q=|E(G)|$ ), the super line graph $\mathscr{L}_{r}(G)$ of index $r$ has the sets of $r$ edges in $G$ as its vertices, and two vertices are adjacent if an edge in one set is adjacent (in $G$ ) to an edge in the other. It follows that $\mathscr{L}_{1}(G)$ is the usual line graph. Figure 3.1 shows an example of a graph $G$ and the graph $\mathscr{L}_{2}(G)$. For simplicity, we denote a set $\{x, y\}$ of edges by $x y$.

Several variations of the definition of a super line graph can be considered. For example, one could form a multigraph by joining two vertices with as many edges as there are adjacencies between the two sets of edges. We call this the super line multigraph. See [3] for some results. Or, we can form the intersection graph of the sets of vertices on the two sets of edges.

The super line graph operator has nice hereditary properties, as the next two results show [1].

THEOREM 3.1. If $G$ is a subgraph of graph $H$, then $\mathscr{L}_{r}(G)$ is an induced subgraph of $\mathscr{L}_{r}(H)$.

THEOREM 3.2. Let $G$ be a graph with $q$ edges. For $r<q / 2, \mathscr{L}_{r}(G)$ is isomorphic to a subgraph of $\mathscr{L}_{r+1}(G)$.

The definition of adjacency in the super line graph $\mathscr{L}_{r}(G)$ implies that if two vertices (say) $S$ and $T$ are nonadjacent, then (with $S$ and $T$ considered as $r$-sets of edges) their 

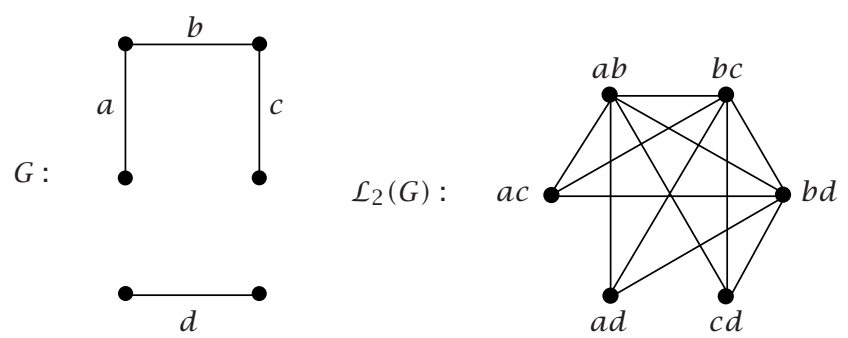

FIGURE 3.1

intersection $S \cap T$ is a set of isolated edges in the subgraph (of $G$ ) of their union $S \cup T$. This leads to our next result [6].

THEOREM 3.3. If $S$ and $T$ are $r$-sets of edges in $G$ such that neither consists entirely of $r$ isolated components of $G$, then the distance between $S$ and $T$ in $\mathscr{L}_{r}(G)$ is 1 or 2 .

Proof. If such $S$ and $T$ are not adjacent in $\mathscr{L}_{r}(G)$, then $G$ contains an edge $e$ adjacent to some edge in $S$ and an edge $f$ adjacent to some edge in $T$. Now let $R$ be any set of $r$ edges of $G$ containing $e$ and $f$. Then both $S$ and $T$ are adjacent to $R$ in $\mathscr{L}_{r}(G)$, and so the distance between them is 2 .

COROLLARY 3.4. If $G$ is a graph in which fewer than $r$ components are isolated edges, then $\mathscr{L}_{r}(G)$ has diameter 1 or 2.

COROLlARY 3.5. For a graph $G$, at most one component of $\mathscr{L}_{r}(G)$ is nontrivial.

3.2. Line completion number. We observe that if $\mathscr{L}_{r}(G)$ is complete, so is $\mathscr{L}_{S}(G)$ for $r \leq s \leq q$. This leads us to define the line completion number $l c(G)$ of graph $G$ to be the minimum index $r$ for which $\mathscr{L}_{r}(G)$ is complete. Clearly, $l c(G) \leq q$. We observe that the only graphs with the line completion number 1 are the stars and $K_{3}$.

A general bound on line completion number is given by the following result [6].

THEOREM 3.6. If $G$ is a graph with $q$ edges and c components, then $l c(G) \leq\lfloor(q+$ c) /2」, and this bound is sharp.

The next theorem [5] describes which graphs have small or large line completion numbers.

THEOREM 3.7. (i) $\ell c(G)=1$ if and only if $G$ is $K_{1, n}$ or $K_{3}$.

(ii) $\ell_{c}(G) \leq 2$ if and only if $G$ does not have $3 K_{2}$ or $2 P_{3}$ as a subgraph.

(iii) $\ell c(G) \leq 3$ if and only if $G$ does not have any of the following as a subgraph: $4 K_{2}$, $K_{2} \cup 2 K_{1,2}, 2 K_{3}, K_{3} \cup P_{4}, K_{3} \cup K_{1,3}, 2 K_{1,3}, K_{1,3} \cup P_{4}$.

(iv) $\ell c(G)=q$ if and only if $G \cong q K_{2}$.

(v) $\ell c(G)=q-1$ if and only if $G \cong P_{3} \cup(q-2) K_{2}$ or $G \cong 2 P_{3} \cup(q-4) K_{2}$.

Line completion numbers of several classes of graphs have been found. We list some of these in the next theorem. See [5] for more details. 
THEOREM 3.8. (i) If $T$ is a tree of order $n \geq 2$, then $\ell c(T) \leq\lfloor n / 2\rfloor$. Furthermore, for any integer $k$ satisfying $1 \leq k \leq\lfloor n / 2\rfloor$, there is a tree $T$ of order $n$ with $\ell c(T)=k$.

(ii) $\ell c\left(K_{n}\right)=(1 / 2)\lfloor n / 2\rfloor(\lfloor n / 2\rfloor-1)+1$.

(iii) $\ell c\left(P_{n}\right)=\ell c\left(C_{n}\right)=\lfloor n / 2\rfloor$.

(iv) $\ell c\left(K_{1}+P_{n}\right)=\ell c\left(K_{1}+C_{n}\right)=\lfloor 2 n / 3\rfloor$.

(v) $\ell c\left(K_{1}+n K_{2}\right)=\lfloor 3 n / 4\rfloor+1$.

Line completion numbers of several other classes of graphs have also been obtained. These classes include hypercubes, ladders, and grids. One class of graphs for which only partial results are known is the class of complete bipartite graphs. We list the known cases in the next theorem.

THEOREM 3.9. (i) For $m=2 r, n=2 s, l c\left(K_{m, n}\right)=r s+1=m n / 4+1$.

(ii) If $m \mid n$, and $m$ is odd, then $l_{c}\left(K_{m, n}\right)=n\left({ }^{2}-1\right) / 4 m$.

(iii) $l c\left(K_{2 r, 2 r q+1}\right)=r^{2} q$.

(iv) $l c\left(K_{2 r, 2 r q+3}\right)=r(r q+1)$ for $q \geq 1$.

(v) $l c\left(K_{2 r+1,2 r+4 k}\right)=(r+k)^{2}$ for $r \geq 4 k^{2}-3 k$.

A complete determination of $l c\left(K_{m, n}\right)$ is still open.

3.3. Cycles in super line graphs. We now describe several interesting results on cycles in super line graphs. Even though super line graphs can be dense, they, in general, do not satisfy well-known sufficient conditions for Hamiltonicity. For any connected graph $G$, however, $\mathscr{L}_{2}(G)$ turns out to be vertex pancyclic, that is, every vertex lies on cycles of length three through the order of the graph. In fact, as we see below, this result is true for many disconnected graphs also. We will state most results without proof. However, to give a flavor of the techniques used, we include a special result with proof.

THEOREM 3.10. If $T$ is a tree with $q \geq 2$ edges, then $\mathscr{L}_{2}(T)$ is pancyclic.

Proof. The proof is by induction on $q$. For $q \leq 4$, it can be easily checked that $\mathscr{L}_{2}(T)$ is complete, which is also true if $T$ is a star. Assume that $T$ is not a star and that $q>4$. Choose a vertex $u$ of $T$ such that $\operatorname{deg}(u)>1$, and every neighbor of $u$, except one, say $v$, is an end vertex. Since $T$ is not a star, such a vertex always exists. Let $S$ be the star with center $u$, and let $T_{v}$ be the subtree of $T$ obtained by removing $u$ and all its neighbors except $v$. Let $s=\operatorname{deg}(u)$ so that $T_{v}$ has $r=q-s$ edges. By our choice of $u$ and $v$, it follows that $r>0$ and $s>1$. If $r=1$, then $\mathscr{L}_{2}(T)$ is complete. If $r=2$ with (say) $E\left(T_{v}\right)=\left\{f_{1}, f_{2}\right\}$, then $\mathscr{L}_{2}(T)-f_{1} f_{2}$ is complete, and $\operatorname{deg}_{\mathscr{L}_{2}(T)} f_{1} f_{2}>1$. Hence $\mathscr{L}_{2}(T)$ is pancyclic. Thus we may assume that $r \geq 3$.

Now the vertex set of $\mathscr{L}_{2}(T)$ is the (disjoint) union of $V\left(\mathscr{L}_{2}(S)\right), V\left(\mathscr{L}_{2}\left(T_{v}\right)\right)$, and the set $W=\left\{a b \mid a \in E\left(T_{v}\right), b \in E(S)\right\}$. Also, $\mathscr{L}_{2}(S)$ is complete and, by the induction hypothesis, $\mathscr{L}_{2}\left(T_{v}\right)$ is pancyclic. Let $C_{v}$ be a spanning cycle of $\mathscr{L}_{2}\left(T_{v}\right)$. Also let $X_{1} X_{2}$ be an edge of $C_{v}$, where $X_{1}=f_{1} e$ and $X_{2}=f_{2} f$, where $f_{1}, e, f_{2}, f \in E\left(T_{v}\right)$ and $f_{1}$ is adjacent to $f_{2}$.

We observe that $|W|=r s$. Since $S$ is a star with $s$ edges, the subgraph of $\mathscr{L}_{2}(T)$ induced by $W$ contains the complete multipartite graph $K_{r, r, \ldots, r}$ as a spanning subgraph. 
Hence, it contains paths of lengths 1 through $r s-1$. We can also assume that the end vertices of each of these paths are $Y_{1}=f_{1} g$ and $Y_{2}=f_{2} h$, where $f_{1}$ and $f_{2}$ are as above, and $g, h \in E(S)$.

Now, in $\mathscr{L}_{2}(T), X_{1}$ and $Y_{2}$ are adjacent, as are $X_{2}$ and $Y_{1}$. Hence we can construct cycles of lengths $\left|C_{v}\right|+j=\left(\begin{array}{l}r \\ 2\end{array}\right)+j$, for $2 \leq j \leq r s$, by identifying the ends of each of the above paths appropriately with those of the path $C_{v}-X_{1} X_{2}$.

Let $C$ be a cycle of length $\left(\begin{array}{l}r \\ 2\end{array}\right)+r s$ so constructed. By our construction, $C$ contains edges from the subgraph of $\mathscr{L}_{2}(T)$ induced by $W$. Also, every vertex of the complete graph $\mathscr{L}_{2}(S)=K_{\left(\begin{array}{c}s \\ 2\end{array}\right)}$ is adjacent to all vertices of $W$. It follows that $C$ can be extended to cycles of each length from $\left(\begin{array}{l}s \\ 2\end{array}\right)+r s+1$ to $\left(\begin{array}{l}s \\ 2\end{array}\right)+r s+\left(\begin{array}{l}r \\ 2\end{array}\right)$.

It remains to show the existence of a cycle of length $\left(\begin{array}{l}r \\ 2\end{array}\right)+1$. For this, choose three adjacent vertices, say, $a_{1} b_{1}, a_{2} b_{2}$, and $a_{3} b_{3}$ on $C_{v}$. Assume that $a_{1}$ is adjacent to $a$, and that $a_{3}$ is adjacent to $b$, for some $a, b \in E\left(T_{v}\right)$ (not necessarily distinct). Choose two adjacent edges $e_{1}$ and $e_{2}$ in $S$ and replace the $P_{3}$ formed by the above three vertices by the $P_{4}$ formed by $a_{1} b_{1}, a e_{1}, b e_{2}, a_{3} b_{3}$ to produce the required cycle. This completes the proof.

Now this result can easily be extended to all connected graphs.

THEOREM 3.11. If $G$ is connected, with $q \geq 2$, then $\mathscr{L}_{2}(G)$ is pancyclic.

Proof. The proof is by induction on the number of cycles in $G$. The base case is covered by the previous theorem. Let $e=u v$ be a cycle edge, and $w$ a new vertex not in $V(G)$. Construct a graph $H=G-e+f$, where $f=u w$. Then $\mathscr{L}_{2}(H)$ is isomorphic to a spanning subgraph of $\mathscr{L}_{2}(G)$. Then $H$ is connected and has fewer cycles than $G$. Hence by the induction hypothesis, $\mathscr{L}_{2}(H)$ is pancyclic. It follows that $\mathscr{L}_{2}(G)$ is pancyclic.

As we stated before, however, a much stronger result than the one given in the above theorem holds. We state this in our next theorem [2].

THEOREM 3.12. If $G$ is a graph with no isolated edges, then $\mathscr{L}_{2}(G)$ is vertex pancyclic.

While it may be possible to extend this theorem to some graphs having isolated edges, it cannot be done for all graphs, even to their nontrivial components. For example, let $G$ be the disjoint union $P_{4} \cup 2 K_{2}$, then the nontrivial component of $\mathscr{L}_{2}(G)$ is the complete multipartite graph $K_{1,1,2,5}$, which clearly is not Hamiltonian, and thus is not pancyclic. However, we believe that, for a graph $G$ with at most one isolated edge, $\mathscr{L}_{2}(G)$ is pancyclic, and that it may even be vertex pancyclic.

Further problems along these lines suggest themselves. For example, find conditions on $G$ under which $\mathscr{L}_{2}(G)$ has isolated vertices but the nontrivial component is Hamiltonian, pancyclic, or vertex-pancyclic. Another open problem is to study $\mathscr{L}_{2}(G)$ in relation to Hamiltonian connectedness and panconnectedness.

3.4. Independence number. Let $M$ be a set of independent edges in a graph $G$. If $A$ and $B$ are $r$-sets of $M$, then $A$ and $B$ are nonadjacent in $\mathscr{L}_{r}(G)$. However, not all pairs of nonadjacent vertices arise in this way. It is also the case that two $r$-sets of edges of $G$ are nonadjacent in $\mathscr{L}_{r}(G)$ if they generate vertex-disjoint subgraphs. What we 
show is that when one considers a set of independent vertices in $\mathscr{L}_{r}(G)$ of maximum order, then with a few exceptional families of graphs, it is produced by a maximum independent set of edges of $G$. We use $\alpha(G)$ and $\alpha^{\prime}(G)$ for the independence number and edge-independence number of $G$, respectively. We also denote the set of all $r$-sets of a set $X$ by $\left(\begin{array}{l}X \\ r\end{array}\right)$.

Our next result [2] gives the independence number of $\mathscr{L}_{r}(G)$ in terms of the edgeindependence number of $G$.

THEOREM 3.13. Let $G$ be a graph with at least $r$ edges. Then the independence number of $\mathscr{L}_{r}(G)$ is

$$
\alpha\left(\mathscr{L}_{r}(G)\right)=\left(\begin{array}{c}
\alpha^{\prime}(G) \\
r
\end{array}\right)
$$

Furthermore, if $S$ is a maximum independent set of vertices in $\mathscr{L}_{r}(G)$, then either

(i) $S=\left(\begin{array}{l}X \\ r\end{array}\right)$ for some maximum independent set $X$ of edges of $G$, or

(ii) $S$ consists of $r+1$ disjoint stars $K_{1, r}$, or

(iii) $r=3$ and the vertices in $S$ are $K_{1,3}$ 's or $K_{3}$ 's.

Proof. If $X$ is a maximum independent set of edges of $G$, then, clearly, $r$-sets of $X$ are independent vertices in $\mathscr{L}_{r}(G)$. Thus,

$$
\alpha\left(\mathscr{L}_{r}(G)\right) \geq\left(\begin{array}{c}
\alpha^{\prime}(G) \\
r
\end{array}\right) .
$$

To prove the reverse inequality, let $V_{1}, V_{2}, \ldots, V_{k}$ be $r$-sets of $E(G)$ which are independent vertices in $\mathscr{L}_{r}(G)$. Also, let $m=$ number of these sets which are matchings in $G$, $\ell=$ number of these sets which are not matchings in $G$, and $h=$ number of edges of $G$ in the union of the $m$ matchings. Clearly, $m \leq\left(\begin{array}{l}h \\ r\end{array}\right)$. Let $U=V_{1} \cup V_{2} \cup \cdots \cup V_{k}$. We observe that if two edges of $U$ are adjacent in $G$, they must belong to the same $V_{i}$ and each such pair is in only one $V_{i}$. Thus, we form an independent set of edges in $G$ by taking the $h$ edges mentioned above, and one of the nonindependent edges of each of the $\ell$ nonmatchings. Hence, $\ell+h \leq \alpha^{\prime}(G)=\alpha^{\prime}$, and therefore,

$$
k=\ell+m \leq \ell+\left(\begin{array}{l}
h \\
r
\end{array}\right) \leq\left(\begin{array}{c}
\ell+h \\
r
\end{array}\right) \leq\left(\begin{array}{c}
\alpha^{\prime} \\
r
\end{array}\right),
$$

from which we have the desired inequality.

To prove the second part, let $S=\left\{V_{1}, V_{2}, \ldots, V_{k}\right\}$ be a maximum independent set in $\mathscr{L}_{r}(G)$ with $k=\left(\begin{array}{c}\alpha^{\prime} \\ r\end{array}\right)$. It follows from (3.3) that

$$
k=\ell+m=\ell+\left(\begin{array}{l}
h \\
r
\end{array}\right)=\left(\begin{array}{c}
\ell+h \\
r
\end{array}\right)=\left(\begin{array}{l}
\alpha^{\prime} \\
r
\end{array}\right) .
$$

If $\ell=0$, then $k=m=\left(\begin{array}{l}h \\ r\end{array}\right)=\left(\begin{array}{l}\alpha^{\prime} \\ r\end{array}\right)$ so that $S$ satisfies (i). If $r=1$, then again all $V_{i}$ 's are matchings so that $\ell=0$. Thus we assume that $\ell>0$ and $r>1$. In this case, $\ell+$ $\left(\begin{array}{l}h \\ r\end{array}\right)=\left(\begin{array}{c}\ell+h \\ r\end{array}\right)$ implies that $h=0$ and $\ell=r+1$. Consequently, $\alpha^{\prime}=r+1$. Moreover, it 
follows that $m=0$, so that no $V_{i}$ is a matching. If any $V_{i}$ is not a star, then it has two independent edges or it is a $K_{3}$. In the first case, one obtains $r+2$ independent edges in $G$, a contradiction. In the second case, it follows that $r=3$ so that each $V_{i}$ is a $K_{3}$ or a $K_{1,3}$.

The above theorem characterizes the maximum independent sets in $\mathscr{L}_{r}(G)$. We observe that more can be said about the structure of $G$ in cases (ii) and (iii), namely, that each additional edge in $G$ must join the center of one star to some vertex in another component.

3.5. Other properties and types of super line graphs. We have described several different structural and other properties of super line graphs. The wealth of results indicates that this is a rich and fertile area for research. Some open problems were listed in the above subsections. Bagga et al. [1, 2, 3, 5, 6] have also studied variations such as super line multigraphs and super line digraphs. However, only some preliminary work has been done for these variations and further explorations are required.

4. Triangle graphs. In this section, we describe another recent generalization of line graphs. As noted in the introduction, the vertices of the line graph can be considered as cliques of order 2, with two being adjacent if they have a $K_{1}$ in common. This concept has been generalized to clique graphs. We will mention the general case in the next section. Here, we consider the special case of triangles.

The triangle graph of $G$, denoted by $\mathscr{T}(G)$, is the graph with vertex set the set of triangles in $G$. Two vertices are adjacent in $\mathscr{T}(G)$ if, as triangles of $G$, they share an edge in common. If $G$ has no triangle, then $\mathscr{T}(G)$ is undefined. A graph $H$ is called a triangle graph if $H \simeq \mathscr{T}(G)$ for some $G$. Otherwise it is called a nontriangle graph.

In [21], the problem of determining necessary and sufficient conditions for a graph to be a triangle graph was raised. In $[7,8]$, there is some recent progress towards a solution to this problem. We describe some of these results below.

4.1. Some classes of triangle graphs. We begin by listing several classes of graphs which are triangle graphs. It is easily seen that $K_{n}$ is a triangle graph since $K_{n}=$ $\mathscr{T}\left(K_{1,1, n}\right)$. Similarly, cycles and paths can be shown to be triangle graphs since $C_{n} \simeq$ $\mathscr{T}\left(W_{n}\right)$, for $n \geq 4$, where $W_{n}$ is the wheel, and $P_{n} \simeq \mathscr{T}\left(W_{n}-e\right)$, where $e$ is a rim edge.

SOME PRODUCTS. $K_{m} \times K_{n}=\mathscr{T}\left(K_{1, m, n}\right), K_{m} \times C_{3}=K_{m} \times K_{3} \simeq \mathscr{T}\left(K_{1, m, 3}\right), K_{m} \times C_{n}=$ $\mathscr{T}\left(\overline{K_{m}} \vee C_{n}\right),(m, n \geq 3)$, and $K_{m} \times P_{n}=\mathscr{T}\left(\overline{K_{m}} \vee P_{n+1}\right)$ are all triangle graphs.

The Platonic graphs. As Figure 4.1 shows, $Q_{3}=\mathscr{T}$ (octahedron). Also, tetrahedron $=K_{4}=\mathscr{T}\left(K_{1,1,4}\right)$ and dodecahedron $=\mathscr{T}$ (icosahedron).

We will see below that the octahedron and the icosahedron are nontriangle graphs.

THEOREM 4.1. A tree $H$ is a triangle graph if and only if $\Delta(H) \leq 3$.

Proof. Observe that a graph that contains $K_{1,4}$ as an induced subgraph is a nontriangle graph. Conversely, if $\Delta(H) \leq 3$, use induction on $|(V(H))|$.

We next describe a necessary condition involving $K_{4}-e$ [8]. 


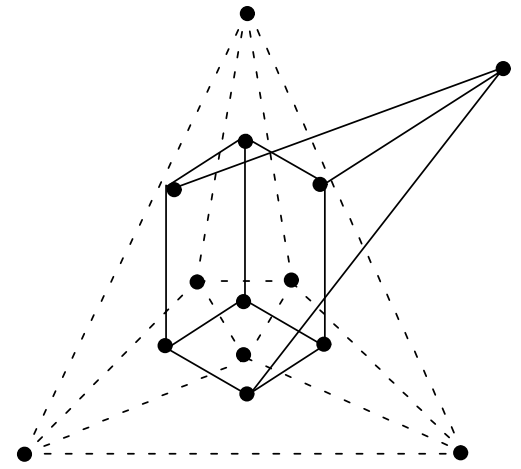

FIGURE 4.1

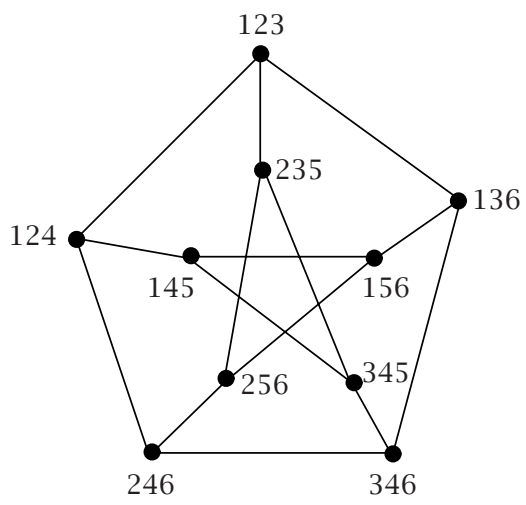

FIGURE 4.2

THEOREM 4.2. If $H$ is a triangle graph with $K_{4}-e$ as an induced subgraph, then there exists a vertex $x$ in $H$ such that $x$ is adjacent to three vertices of one triangle of $K_{4}-e$ and nonadjacent to the fourth vertex.

COROLLARY 4.3. For any $n \geq 4, K_{n}-e$ is not a triangle graph.

COROLLARY 4.4. The octahedron and the icosahedron are nontriangle graphs.

4.2. Triangle labeling of a graph. We next consider another class of graphs that strictly includes the class of triangle graphs. A triangle labeling of a graph is defined to be a mapping $f: V(H) \rightarrow N^{3}$ (triples of positive integers) such that $x y \in E(H)$ if and only if $|f(x) \cap f(y)|=2$.

Figure 4.2 shows a triangle labeling of the Petersen graph. It can be shown by a direct argument that the Petersen graph is not a triangle graph.

THEOREM 4.5. A triangle graph admits a triangle labeling. The converse is not true.

This leads us to define some new classes of graphs as follows. Let $\mathscr{L}=$ the set of line graphs, $\mathscr{L}_{s}=$ the set of induced subgraphs of line graphs, $\mathscr{T}=$ the set of triangle graphs, 
$\mathscr{T}_{s}=$ the set of induced subgraphs of triangle graphs, and $\mathscr{T}_{l}=$ the set of graphs that admit a triangle labeling. We then have the following result [8].

THEOREM 4.6. (i) $\mathscr{L}=\mathscr{L}_{s}$.

(ii) $\mathscr{T} \subset \mathscr{T}_{s}$.

(iii) $\mathscr{T}_{s}=\mathscr{T}_{l}$.

For some more necessary conditions on triangle graphs, and some other classes of triangle graphs, we refer the reader to [8]. The general characterization of triangle graphs is an open problem and further explorations in this area should be undertaken.

4.3. Open problems. We conclude our discussion of triangle graphs by listing a few problems and directions for more investigations in this area. Of course, it would be nice to have a characterization of triangle graphs. As mentioned above, [8] has made some progress in this direction by obtaining a number of necessary conditions, including a forbidden subgraph condition. We saw earlier that several product graphs are triangle graphs. Similar questions can also be asked for other products; in particular, for which graphs $G$ is the Cartesian product $K_{n} \times G$ a triangle graph? We observe that $\mathscr{T}\left(K_{5}\right)=$ $L\left(K_{5}\right)$. One wishes to characterize $G$ for which $\mathscr{T}(G) \simeq L(G)$. Similarly, when is $\mathscr{T}(G) \simeq$ $G$ ? Let $G_{1}$ and $G_{2}$ be graphs in which each edge belongs to a triangle. Under what conditions does $\mathscr{T}\left(G_{1}\right) \simeq \mathscr{T}\left(G_{2}\right)$ imply that $G_{1} \simeq G_{2}$ ? Motivated by the definition of line completion number defined in Section 3.2, we can define the triangle completion number, $t c(G)$, to be the minimum number of edges to be added to $G$ so that the resulting graph becomes a triangle graph. As a first step, one wants bounds for $t c(G)$ for a given graph $G$.

5. Some more generalizations. It was mentioned at the beginning of Section 4 that triangle graphs are a special case of clique graphs, a more general variation on line graphs. In this section, we briefly describe clique graphs and some other variations. For more details and results, we refer the reader to Prisner [22]. We observe that by a clique of a graph $G$, we mean a complete subgraph of $G$. Some authors (including Prisner [22]) use the term simplex for a complete subgraph and clique for inclusion-maximal simplices. Since an edge is a clique of order 2, a generalization of the line graph $L(G)$ of a graph $G$ is obtained when one considers all cliques of $G$ of a fixed order. Depending on how one defines adjacency, several variations are possible, as we see below. The following definitions are from [22].

(i) The $k$-Gallai graph $\varphi_{k}(G)$ has all $K_{k}$ 's in $G$ as vertices, with two adjacent if their union induces a $K_{k+1}-e$. Observe that $\mathscr{G}_{1}(G)=\bar{G}$.

(ii) The $k$-in- $m$ graph $\Phi_{k, m}$ has all $K_{k}$ 's in $G$ as vertices, with two adjacent if they lie in a common $K_{r}$ for some $r \leq m$.

(iii) The $k$-line graph $L_{k}(k \geq 2)$ has all $K_{k}$ 's in $G$ as vertices, with two adjacent if their intersection is a $K_{k-1}$. We observe that $L_{k}(G)$ is the edge-disjoint union of $\Phi_{k, k+1}(G)$ and $\varphi_{k}(G)$.

(iv) The $k$-overlap clique graph $C_{k}(G)$ of $G$ has all maximal cliques of $G$ as vertices, with two adjacent whenever their intersection contains at most $k$ vertices. 
(v) The cycle graph $C y(G)$ of a graph $G$ has all induced cycles of $G$ as vertices, with two adjacent if they share some common edge.

For more such generalizations and their properties, we refer the reader to [22].

6. Conclusion. In this brief survey, we have described line graphs and their generalizations. Many of the generalizations are well known and well studied. Others are more recent. For all generalizations, a number of problems and areas of further study have been presented. This in an active area of research. We have included a set of references which have been cited in our description. These references are just a small part of the literature, but they should provide a good start for readers interested in this area.

ACKNOWLEDGMENT. The author wishes to express his gratitude to all of his coauthors listed in the references. The author also thanks the referees for their helpful comments.

\section{REFERENCES}

[1] J. S. Bagga, L. W. Beineke, and B. N. Varma, Super line graphs and their properties, Combinatorics, Graph Theory, Algorithms and Applications (Beijing, 1993), World Scientific Publishing, New Jersey, 1994, pp. 1-6.

[2] __ Independence and cycles in super line graphs, Australas. J. Combin. 19 (1999), 171-178.

[3] _ The super line graph $\mathscr{L}_{2}$, Discrete Math. 206 (1999), no. 1-3, 51-61.

[4] K. J. Bagga and M. R. Vasquez, The super line graph $\mathscr{L}_{2}$ for hypercubes, Congr. Numer. 93 (1993), 111-113.

[5] K. S. Bagga, L. W. Beineke, and B. N. Varma, The line completion number of a graph, Graph Theory, Combinatorics, and Algorithms, Vol. 1, 2 (Kalamazoo, Mich, 1992), WileyInterscience, New York, 1995, pp. 1197-1201.

[6] _ Super line graphs, Graph Theory, Combinatorics, and Algorithms, Vol. 1, 2 (Kalamazoo, Mich, 1992), Wiley-Interscience, New York, 1995, pp. 35-46.

[7] R. Balakrishnan, Triangle graphs, Graph Connections (Cochin, 1998), Allied Publishers, New Delhi, 1999, p. 44.

[8] R. Balakrishnan, J. Bagga, R. Sampathkumar, and N. Thillaigovindan, Triangle graphs, preprint, 2002.

[9] R. Balakrishnan and K. Ranganathan, A Textbook of Graph Theory, Universitext, SpringerVerlag, New York, 2000.

[10] M. Behzad and G. Chartrand, Total graphs and traversability, Proc. Edinburgh Math. Soc. (2) 15 (1966/1967), 117-120.

[11] L. W. Beineke, Characterizations of derived graphs, J. Combinatorial Theory 9 (1970), 129135.

[12] H. J. Broersma and C. Hoede, Path graphs, J. Graph Theory 13 (1989), no. 4, 427-444.

[13] G. Chartrand, On hamiltonian line-graphs, Trans. Amer. Math. Soc. 134 (1968), 559-566.

[14] G. Chartrand, H. Hevia, E. B. Jarrett, F. Saba, and D. W. VanderJagt, Subgraph distance and generalized line graphs, Graph Theory, Combinatorics, Algorithms, and Applications (San Francisco, Calif, 1989), SIAM, Philadelphia, 1991, pp. 266-280.

[15] A. G. Chetwynd, Total colourings of graphs - a progress report, Graph Theory, Combinatorics, and Applications, Vol. 1 (Kalamazoo, Mich, 1988), Wiley-Interscience, New York, 1991, pp. 233-244.

[16] F. Harary, Graph Theory, Addison-Wesley, Massachusetts, 1969.

[17] F. Harary and R. Z. Norman, Some properties of line digraphs, Rend. Circ. Mat. Palermo (2) 9 (1960), 161-168. 
[18] R. L. Hemminger and L. W. Beineke, Line graphs and line digraphs, Selected Topics in Graph Theory (W. B. Lowell and R. J. Wilson, eds.), Academic Press, New York, 1978, pp. 271-305.

[19] J. Krausz, Démonstration nouvelle d'une théorème de Whitney sur les réseaux, Mat. Fiz. Lapok 50 (1943), 75-85 (Hungarian).

[20] J. Mitchem, Hamiltonian and Eulerian properties of entire graphs, Graph Theory and Applications (Proc. Conf., Western Michigan Univ., Kalamazoo, Mich, 1972; Dedicated to the Memory of J. W. T. Youngs), Lecture Notes in Math., vol. 303, Springer, Berlin, 1972, pp. 189-195.

[21] S. D. Monson, N. J. Pullman, and R. Rees, A survey of clique and biclique coverings and factorizations of (0,1)-matrices, Bull. Inst. Combin. Appl. 14 (1995), 17-86.

[22] E. Prisner, Graph Dynamics, Pitman Research Notes in Mathematics Series, vol. 338, Longman, Harlow, 1995.

[23] A. C. M. van Rooij and H. S. Wilf, The interchange graph of a finite graph, Acta Math. Acad. Sci. Hungar. 16 (1965), 263-269.

[24] H. Whitney, Congruent graphs and the connectivity of graphs, Amer. J. Math. 54 (1932), $150-168$.

Jay Bagga: Department of Computer Science, Ball State University, Muncie, IN 47306, USA

E-mail address: jbagga@bsu . edu 


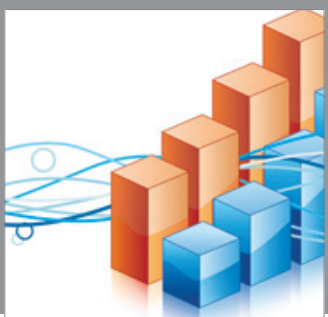

Advances in

Operations Research

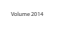

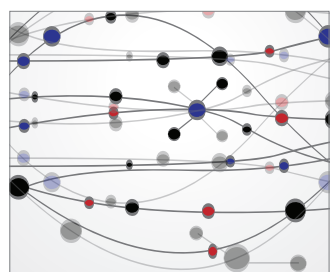

\section{The Scientific} World Journal
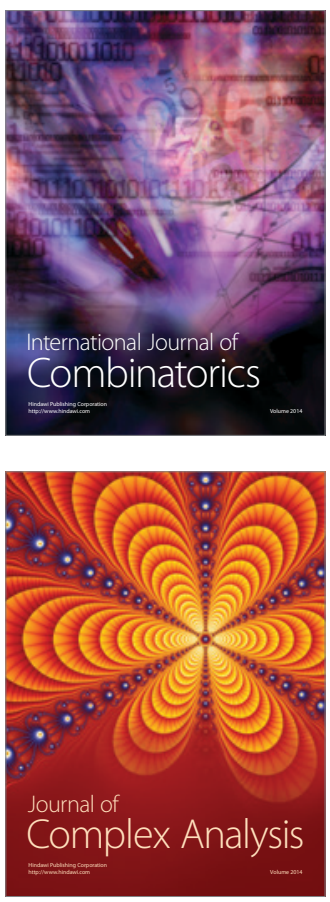

International Journal of

Mathematics and

Mathematical

Sciences
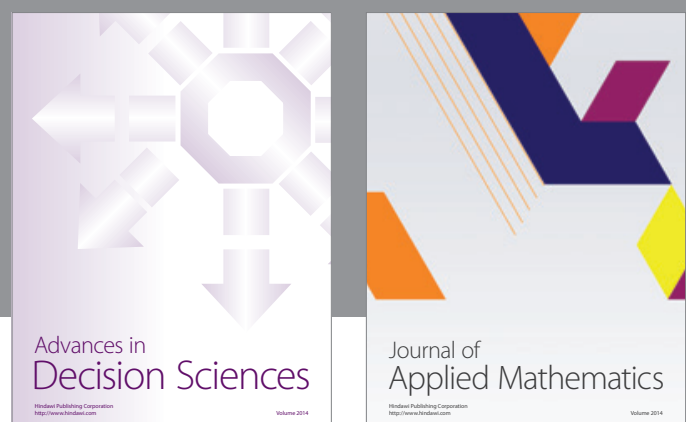

Journal of

Applied Mathematics
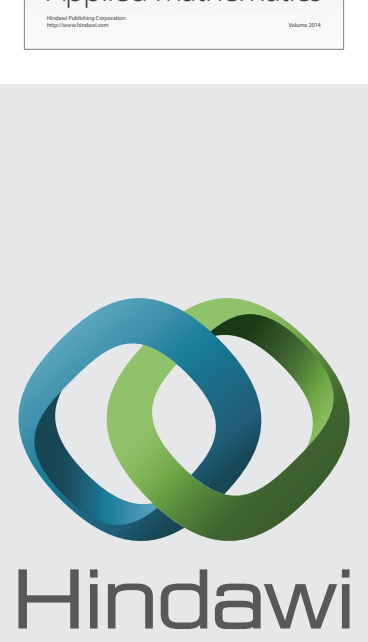

Submit your manuscripts at http://www.hindawi.com
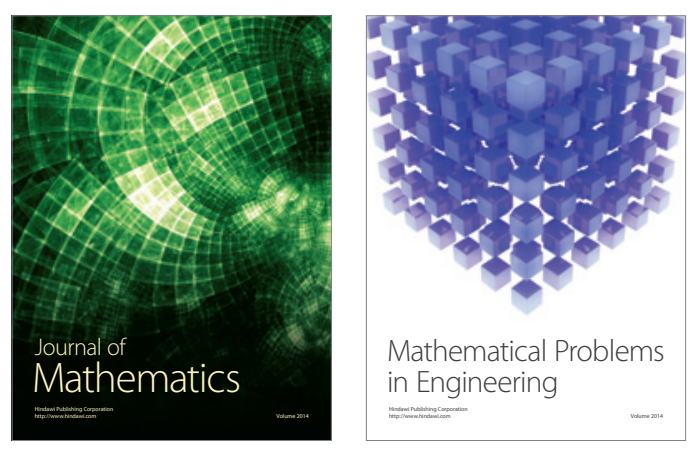

Mathematical Problems in Engineering
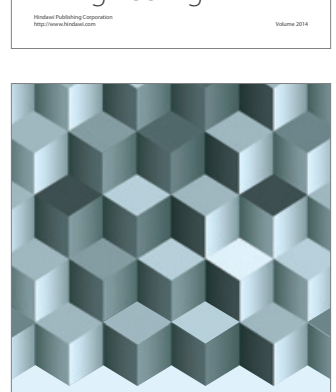

Journal of

Function Spaces
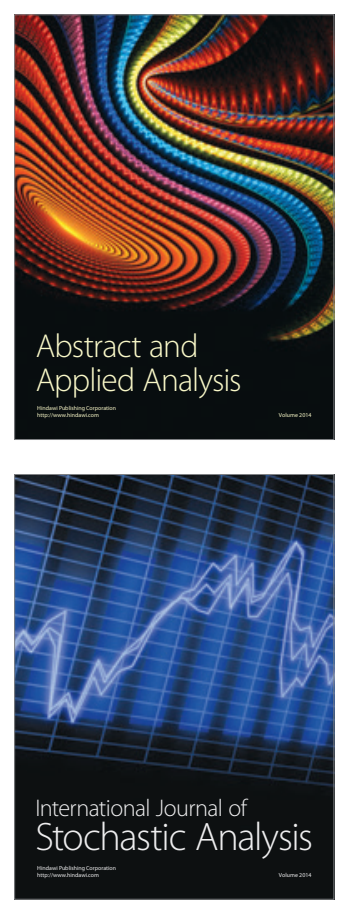

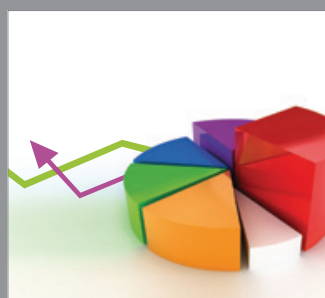

ournal of

Probability and Statistics

Promensencen
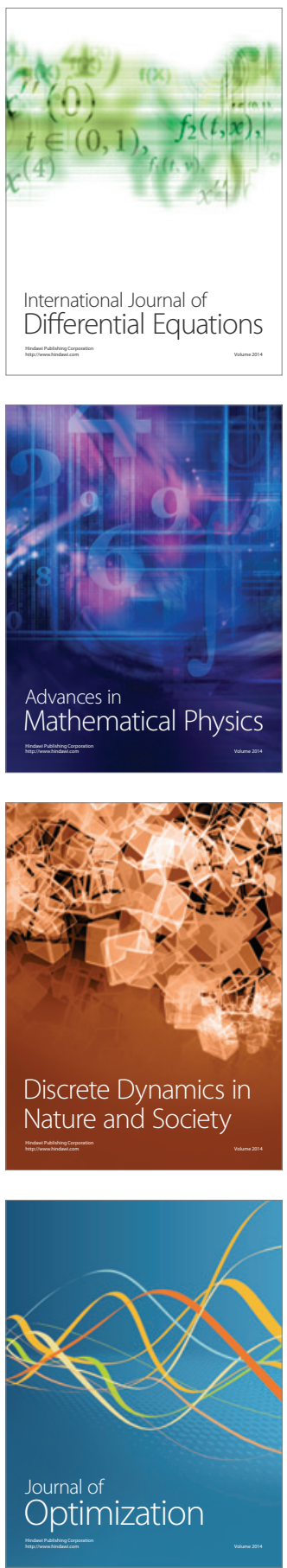